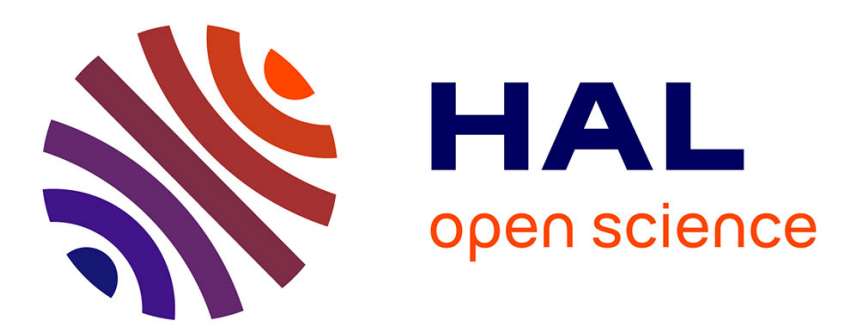

\title{
Human-Intelligent System Shared Control Strategy with Conflict Resolution
}

Shriram Jugade, Alessandro Corrêa Victorino, Véronique Cherfaoui

\section{To cite this version:}

Shriram Jugade, Alessandro Corrêa Victorino, Véronique Cherfaoui. Human-Intelligent System Shared Control Strategy with Conflict Resolution. 14th International Conference on Control and Automation (ICCA 2018), Jun 2018, Anchorage, Alaska, United States. pp.686-691, 10.1109/ICCA.2018.8444218 . hal-01943609

\section{HAL Id: hal-01943609 \\ https://hal.science/hal-01943609}

Submitted on 13 Jul 2021

HAL is a multi-disciplinary open access archive for the deposit and dissemination of scientific research documents, whether they are published or not. The documents may come from teaching and research institutions in France or abroad, or from public or private research centers.
L'archive ouverte pluridisciplinaire HAL, est destinée au dépôt et à la diffusion de documents scientifiques de niveau recherche, publiés ou non, émanant des établissements d'enseignement et de recherche français ou étrangers, des laboratoires publics ou privés. 


\title{
Human-Intelligent System Shared Control Strategy with Conflict Resolution
}

\author{
Shriram C Jugade ${ }^{1}$, Alessandro C Victorino ${ }^{2}$ and Véronique B Cherfaoui ${ }^{1}$
}

\begin{abstract}
Shared control strategies play a very important role in the Human-Intelligent system collaboration thus bridging the gap between them. It gives the scope to bring out the best in human and intelligent system (IntelSys). Different successful shared control strategies developed in the past, limit the scope of collaboration or put restrictions on the independent human and IntelSys behavior. In this paper, we have presented a new generic shared control framework by introducing the idea of a separate fusion system with the objective to blend human-IntelSys individual control inputs. Only fusion system has the direct control of the plant system. The fusion system is built with a new approach, which is based on quantifying and analyzing the difference in the individual control inputs which is termed as 'Conflict'. Shared control is achieved by resolving this conflict which is expressed in the form of a linear quadratic cost function. The shared controller is designed as an adaptive linear quadratic regulator. The generic nature of this framework covers various types of use cases with respect to the admissibility or correctness of the individual control inputs. The shared control strategy is validated on the simulated inverted pendulum system in the simulated environment using Simulink software.
\end{abstract}

\section{INTRODUCTION}

The reliance of humans on the intelligent systems (IntelSys) to perform several functions is on increase. Thus, we can see the transition of IntelSys going from remotely controlled by humans to achieving full autonomy. The IntelSys still require human assistance to perform complex tasks smoothly. Human and IntelSys have different abilities concerning their performance especially in the application of vehicle driving which is the area of interest of our lab research. Humans are more intelligent, experienced and adaptive to the external situations. On other side, IntelSys are more precise and consistent over a long period. The combination of both these qualities are essential to evolve the way we drive the vehicles.

The ability of the IntelSys to handle things or perform tasks is very different as compared to humans. The main task of shared control algorithms is to handle the difference in the human and IntelSys behavior. One of the ways to achieve shared control is to make the IntelSys behavior adaptive i.e. it can lead or follow depending on human intentions and task conditions [3], [4]. The IntelSys behavior can also be

*This project has received funding from the European Commission under the H2020 Grant agreement ITEAM No. 675999 and by LABEX MS2T, ROBOTEX

${ }^{1}$ Sorbonne universités, Université de Technologie de Compiègne, CNRS, Heudiasyc UMR 7253, CS 60319; 60203 Compigne Cedex, France. Email: shriram.jugade/veronique.cherfaoui@ hds.utc.fr

${ }^{2}$ Department of Mechanical Engineering, Universidade Federal de Minas Gerais (UFMG), Av Antonio Carlos 6627, Belo Horizonte, Minas Gerais, Brazil. Email: avictorino@ufmg.br divided into two parts, adaptive (considers human intention) and autonomous. The shared control combines these two behaviors as shown in [5].

Shared conttrol has been applied to complex tasks such as cooperative vehicle driving (ADAS). For e.g. in [6], the shared control is developed for the longitudinal vehicle control using differential game theory. Human and ADAS cooperate with each other by working towards a common goal. In real practice, human driver can not work in such a cooperative manner. Another strategy used is to switch control between human and IntelSys as shown in [1],[7],[8], where human acts as a supervisor. IntelSys has the default control but the human switches control in case of any IntelSys error. In [2], the final control input is formed through the linear combination of human and IntelSys inputs. The coefficient for the linear combination is varied according to different scenarios.

In the proposed approach, we have developed a shared control framework which will have all the benefits of past strategies like switching control, role adaptation, blending individual control inputs. We have introduced a fusion system which will separately perform the task of blending the inputs. This will not put any restrictions on the human and IntelSys behavior. The term fusion used in the paper has a different meaning as compared to its use in sensor data fusion. Fusion system analyzes the difference in the human and IntelSys strategies through the concept of conflict. Shared control is achieved through the conflict resolution.

The paper is organized as follows: Section II firstly introduces the concept of fusion system and the new quantity 'conflict'. The realization of fusion via conflict resolution is explained. The conflict resolution is then presented as an optimal control problem considering conflict as a system state. State space model for conflict is derived. Section III firstly introduces the control problem statement which also defines the shared controller as adaptive linear quadratic regulator (ALQR). The negative effect of admissibility of individual control inputs on the conflict resolution is explained. To incorporate this effect, the shared controller is enhanced. Section IV present the simulation setup in Simulink, simulation results and analysis. Section V present the conclusions with respect to the shared control strategy and simulation results and analysis.

\section{Shared Control Framework}

\section{A. Fusion System Concept}

Fusion of the inputs from human and IntelSys needs to be a separate and independent task to have an efficient shared 
control. Consider an example of shared steering control in a car. If both human and IntelSys are given the direct control of the steering wheel, the individual control inputs will be naturally fused. But the shared steering controller has to be built in the IntelSys itself. This will unnecessarily complicate the things and will not provide efficient shared control. In the presented strategy, neither human nor IntelSys are given direct control of the multi-actuated plant. A separate fusion system is introduced as shown in the Fig.1. Fusion system will blend the individual inputs to provide a final control input to the plant. Another advantage of having a separate system for fusion is that it can be used for different types of shared control applications.

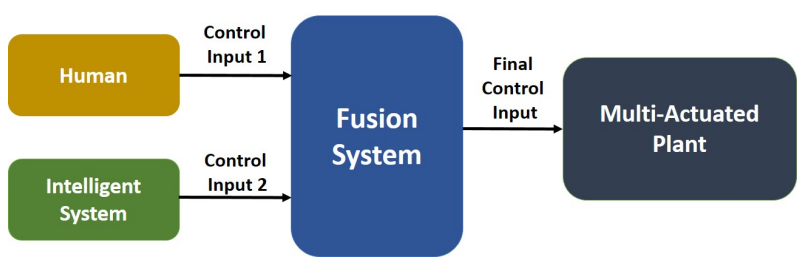

Fig. 1. Fusion System Concept

\section{B. Fusion via Conflict Resolution}

Human and IntelSys have different control strategies i.e. their intended state trajectories of the plant are different. This can be viewed as a conflict between human and IntelSys. The fusion system needs to quantify it and consider this conflict for its function. Hence, we define conflict at a given time instant as the difference between individual control inputs: if $u_{1}(t)$ and $u_{2}(t)$ are the control inputs of human and IntelSys respectively then, the conflict is given as:

$$
\text { Conflict }(t)=u_{1}(t)-u_{2}(t)
$$

The overall system consisting of human, IntelSys, fusion system and the plant is a closed loop. Hence, given a nonzero initial conflict i.e. Conflict $(t=0) \neq 0$, the goal of the shared control strategy is to regulate $\operatorname{Conflict}(t=0)$ to 0 in a given finite interval of time $[0, \mathrm{~T}]$, in the closed loop cited above. This regulation of the Conflict is done by the fusion system, and it is called here the "Conflict Resolution", as described in the next section.

\section{Conflict Resolution: An optimal control problem}

Consider Fig. 2, which shows the global methodology including fusion system in detail. Conflict acts as an input to the shared controller system. In a global automatic control view, the shared controller will compute the final control input in order to regulate the conflict to zero, in a closed loop strategy, where the conflict at time $(t+1)$ is related to the final control input at time $t$.

In order to design the shared controller, it is necessary to derive a model for the conflict as a state system representation, with the final control input as its input and the conflict as its state. This is described in the next section. The conflict model is derived from the global methodology and is represented as shown in Fig. 3.

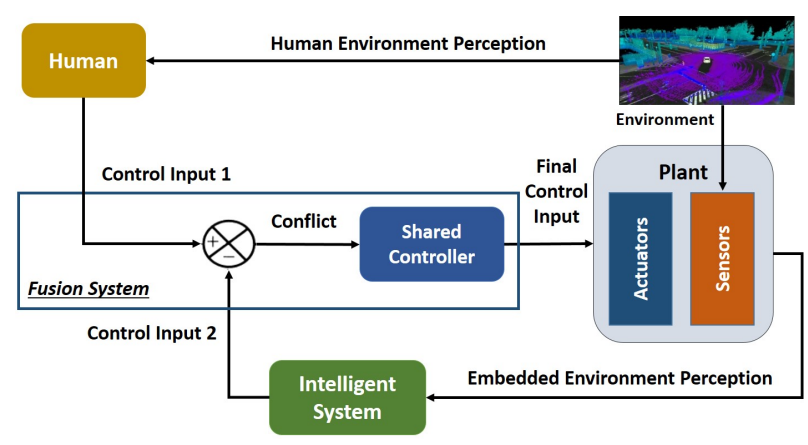

Fig. 2. Global Methodology for Shared Control

\section{Conflict Modeling}

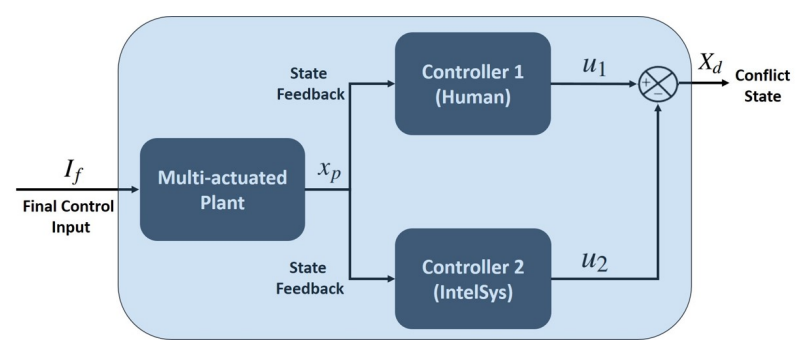

Fig. 3. Conflict Plant/System comprising various components

To apply optimal control techniques for conflict resolution, a mathematical model need to be built from the abstract conflict plant/system. Fig. 3 shows the various components from the global methodology present in the conflict plant/system. The mathematical model built from this plant is termed as Conflict model, and it is derived here for a generic abstract plant (it will be applied to a concrete plant example in the validation section).

Consider the multi-actuated plant state space model as:

$$
\dot{x}_{p}=A_{p} x_{p}+B_{p} I_{f}
$$

where $x_{p} \in \mathbb{R}^{n}$ is the plant state and $I_{f} \in \mathbb{R}^{m}$ is the final control input. The output of the system is considered to be same as state values. Consider the following nonlinearmodel equations, where $\left(u_{1}, u_{2}\right) \in \mathbb{R}^{m}$ are the control inputs of human and IntelSys respectively.

$$
u_{1}=f_{1}\left(x_{p}\right), u_{2}=f_{2}\left(x_{p}\right)
$$

These control models can be linearized as:

$$
u_{1}=M_{1} x_{p}, \quad u_{2}=M_{2} x_{p}
$$

Conflict state $X_{d} \in \mathbb{R}^{m}$ can be given as:

$$
X_{d}=u_{1}-u_{2}=\left(M_{1}-M_{2}\right) x_{p}
$$

Differentiating Equation 5:

$$
\dot{X}_{d}=\left(M_{1}-M_{2}\right) \dot{x_{p}}=\left(M_{1}-M_{2}\right)\left(A_{p} x_{p}+B_{p} I_{f}\right)
$$

Equation 5 can be modified as:

$$
\left(M_{1}-M_{2}\right)^{T} X_{d}=\left(M_{1}-M_{2}\right)^{T}\left(M_{1}-M_{2}\right) x_{p}
$$


Without loss of generalization, we can assume the matrix $\left(M_{1}-M_{2}\right)^{T}\left(M_{1}-M_{2}\right)$ to be invertible. In the case where this matrix is non-invertible, it can be replaced by nearest invertible matrix. Hence, Equation 7 can be written as:

$$
x_{p}=\left(\left(M_{1}-M_{2}\right)^{T}\left(M_{1}-M_{2}\right)\right)^{-1}\left(M_{1}-M_{2}\right)^{T} X_{d}
$$

Let $M=\left(M_{1}-M_{2}\right)$. Substituting for $x_{p}$ from Equation 8 in Equation 6:

$$
\begin{gathered}
\dot{X}_{d}=M A_{p}\left(M^{T} M\right)^{-1}(M)^{T} X_{d}+M B_{p} I_{f} \\
\text { III. Shared CONTROL StrateGy }
\end{gathered}
$$

\section{Shared Control Strategy}

\section{A. Optimal Control Problem Statement}

The global methodology can be represented as a closed loop system containing the conflict plant model and the shared controller. The situations and the individual control strategies keep changing because of which optimization has to be performed repeatedly with the updated model parameters. Hence, the shared control is considered to be an adaptive LQR control to update the control gain according to the changes in the behavior of human and IntelSys. In the past, different strategies for adaptive LQR have been developed for different applications (for e.g. [9]).

The cost function to be used for the optimization is given in the linear quadratic form as:

$$
\operatorname{Cost}=\int_{0}^{\infty} X_{d}^{T} Q_{d} X_{d}+I_{f}^{T} R_{d} I_{f}
$$

The optimal final control input is given by solving the LQR problem:

$$
I_{f}=-K X_{d}
$$

where $K$ is the optimal gain, $X_{d}$ is the conflict state. Then, associating the conflict model (Equation 9) and the control optimization (Equations 10, 11), Conflict $(t)$ is regulated to zero in a limited time. This is validated by the results presented in Section IV. Let us consider now the case near to the reality, where the models (given by Equation 4) are inconsistent or inadmissible. This is discussed in next subsections.

\section{B. Admissibility of Individual Control Inputs}

The performance of the shared controller is dependent on the accuracy of the conflict state space model. Behavioral models of human and IntelSys are included in the conflict state space model. These behavioral models are admissible because their output (control input to the plant) is admissible. In reality, the human and IntelSys may behave differently as compared to their behavioral models. This difference (unknown) can be represented by a mismatch between actual behavioral model and that considered in the conflict state space model. Also, the actual control inputs from human and IntelSys may be admissible or inadmissible (known). Since the behavioral models considered in the conflict model are admissible, the inadmissible behavior of either human or IntelSys or both can be represented as a behavioral model
TABLE I

CONTROL INPUT ADMISSIBILITY AND BEHAVIORAL MODEL MATCH

\begin{tabular}{|c|c|c|}
\hline Use Case & Control Inputs & Model mismatch? \\
\hline 1 & Inadmissible & Yes \\
\hline 2 & Admissible & Yes \\
\hline 3 & Admissible & No \\
\hline
\end{tabular}

mismatch. The relation between admissibility of individual control inputs and behavioral model mismatch is shown in Table I.

With respect to conflict resolution, the inadmissibility in the individual control inputs is critical for the performance of shared controller and it will fail to resolve the conflict between human and IntelSys if not incorporated in the strategy. The fusion system should work even if either human or IntelSys or both provide inadmissible control inputs. Hence, necessary enhancements in the shared controller design are required.

\section{Enhanced Shared Controller}

Consider the use case where human is providing inadmissible control input. This can be expressed as the change in the human behavior leading to the human model mismatch with respect to the conflict model. Let the actual human behavioral model and the model used by the shared controller be given as:

$$
u_{1}=M_{1} x_{p}, \quad \hat{u}_{1}=\hat{M}_{1} x_{p}
$$

where $u_{1}$ and $\hat{u}_{1}$ are the actual and predicted control inputs respectively. Let $X_{d}$ and $\hat{X}_{d}$ be the actual and predicted conflict states respectively which are given as:

$$
\begin{aligned}
& X_{d}=u_{1}-u_{2}=\left(M_{1}-M_{2}\right)\left(x_{p}\right) \\
& \hat{X}_{d}=\hat{u}_{1}-u_{2}=\left(\hat{M}_{1}-M_{2}\right)\left(x_{p}\right)
\end{aligned}
$$

Let the actual conflict model (unknown) be:

$$
\dot{X}_{d}=A_{d} X_{d}+B_{d} I_{f}
$$

Let the conflict model used by shared controller be:

$$
\dot{\hat{X}}_{d}=\hat{A}_{d} \hat{X}_{d}+\hat{B}_{d} I_{f}
$$

where $X_{d}$ and $\hat{X}_{d}$ are the actual and predicted conflict states respectively.

The final control input was given as shown in Equation 11, rewritten here:

$$
I_{f}=-K X_{d}
$$

The optimal gain calculated using $\hat{A}_{d}, \hat{B}_{d}$. Hence, the eigenvalues of $\left(A_{d}-B_{d} K\right)$ may not be negative. Instead, let us calculate the final control input as follows:

$$
I_{f}=-K \hat{X}_{d}
$$

This implies that the eigenvalues of $\hat{A}_{d}-\hat{B}_{d} K$ are negative and $\hat{X}_{d}$ is stable. Thus, the shared controller will switch from the use of $X_{d}$ to $\hat{X}_{d}$ in the presence of any individual control 


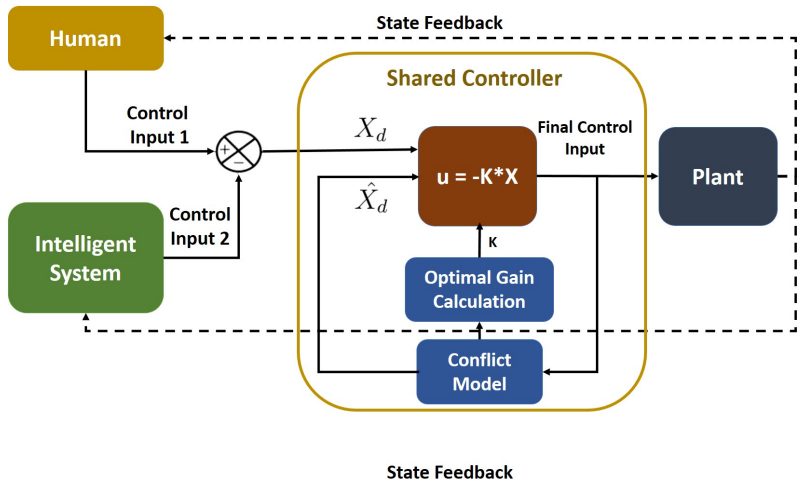

Fig. 4. Enhanced Shared Controller

input admissibility. The enhanced shared controller is shown in Fig.4. Consider the following equations:

$$
\begin{aligned}
& X_{d}=\left(M_{1}-M_{2}\right) x_{p}=M x_{p} \\
& \hat{X}_{d}=\left(\hat{M}_{1}-\hat{M}_{2}\right) x_{p}=\hat{M} x_{p}
\end{aligned}
$$

where $M$ is the actual model coefficient and $\hat{M}$ is the coefficient used in the conflict model. Since we have assumed $M^{T} M$ and $\hat{M}^{T} \hat{M}$ are invertible, Equation 19 and Equation 20 can be written as:

$$
\begin{aligned}
& x_{p}=\left(M^{T} M\right)^{-1} M^{T} X_{d} \\
& x_{p}=\left(\hat{M}^{T} \hat{M}\right)^{-1} \hat{M}^{T} \hat{X}_{d}
\end{aligned}
$$

Comparing the Equations 21 and 22, we can say that stability of $\hat{X}_{d}$ implies stability of $X_{d}$. Hence, the shared controller is enhanced by the inclusion of Equation 18. In brief, $I_{f}$ is calculated using $X_{d}$ or $\hat{X}_{d}$ depending on the admissibility of the individual control inputs. The above enhancement can be applied to other use cases too which are: control input of IntelSys is inadmissible, control inputs of both human and IntelSys are inadmissible.

\section{Simulation Results And Discussion}

The shared control framework had to be validated on a plant/system with a well-known behavior which will help the validation process. Hence, inverted pendulum system ${ }^{1}$ is considered for this purpose as shown in Fig. 5.

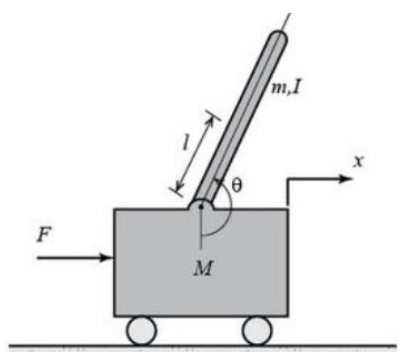

Fig. 5. Inverted Pendulum Configuration

${ }^{1}$ http://ctms.engin.umich.edu/CTMS/index.php?aux=Home
Following is the inverted pendulum model used for the simulation ${ }^{1}$. The input to the inverted pendulum is force. $x$ is the displacement and $\phi$ is the angle deviation from the vertical position of the pendulum. Pendulum angle from vertical (down) is given by $\theta$ and $\theta=\phi+\pi$. Applying the Newton's law, and considering numerical constant values for the parameters $\mathrm{M}, \mathrm{m}, \mathrm{l}$ and $\mathrm{g}$, the model is given by:

$$
\left[\begin{array}{c}
\dot{x} \\
\dot{\phi} \\
\ddot{x} \\
\ddot{\phi}
\end{array}\right]=\left[\begin{array}{cccc}
0 & 1 & 0 & 0 \\
0 & -0.1818 & 2.673 & 0 \\
0 & 0 & 0 & 1 \\
0 & -0.4545 & 31.18 & 0
\end{array}\right]\left[\begin{array}{c}
x \\
\phi \\
\dot{x} \\
\dot{\phi}
\end{array}\right]+\left[\begin{array}{c}
0 \\
1.1818 \\
0 \\
0.4545
\end{array}\right] I_{f}
$$

The main goal is to bring the state to zero by considering control inputs of the human and IntelSys. The final control input is calculated by the fusion between Human and IntelSys using the fusion methodology described in the Section II. All the components in the global methodology are simulated in Matlab/Simulink. The fusion system is expected to provide final control input which is not only optimal but also admissible since there is a direct relation between the conflict resolution and admissibility of final control input. Hence, the selection of inverted pendulum (unstable system) is very beneficial. Any inadmissibility in the final control input will make the system unstable. The performance of the fusion system is validated by comparing it with the individual performances of human and IntelSys controllers as shown in the simulation setup in Fig. 6. Q and R matrices for the shared controller can be selected depending on the requirement of the conflict resolution.

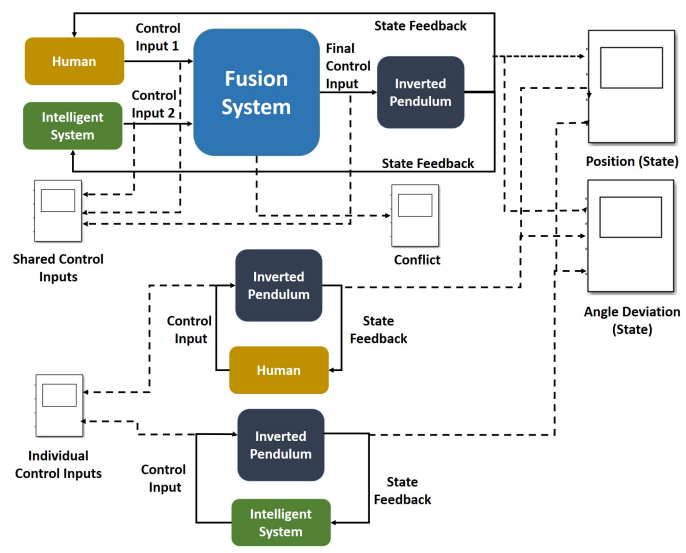

Fig. 6. Simulation setup in Simulink software

High level use cases for the validation are shown in Table II. Inadmissibility is introduced in the human and IntelSys control inputs by modifying the control model parameters. Since the human and IntelSys are simulated as optimal controllers (LQR), inadmissibility is introduced by modifying the optimal gain. Parameters used in the cost function for conflict resolution are: $Q_{d}=10$ and $R_{d}=7$ which are fixed using manual tuning.

Use case 1:

Human control model parameters: $Q_{h}=50 I_{4 \times 4}, R_{h}=2$ 
TABLE II

Use CASES For the VAlidation of Shared Control Strategy

\begin{tabular}{|c|c|c|c|}
\hline Case & Human Input & IntelSys Input & Conflict Model \\
\hline 1 & Admissible & Admissible & Match \\
\hline 2 & Admissible & Admissible & Mismatch \\
\hline 3 & Inadmissible & Admissible & Mismatch \\
\hline 4 & Inadmissible & Inadmissible & Mismatch \\
\hline
\end{tabular}

IntelSys control model parameters: $Q_{r}=2 I_{4 \times 4}, R_{r}=10$ In this type of use case, the shared controller uses the actual conflict state value for the calculation of final control input. Fig. 7 shows the conflict profile. The conflict value is reduced to zero and remains steady at the zero level. The time required for the conflict state to come to zero is dependent on the Q and R matrices used by the Shared controller. Fig. 8 show the inverted pendulum state profiles for shared and independent control by human and IntelSys. The difference in the control behavior of human and IntelSys can be seen through the difference in the state profiles. The nature of the state profile of shared control is not only dependent on the human-IntelSys control behavior but also on the $\mathrm{Q}$ and $\mathrm{R}$ matrices used by the shared controller. The state profile in the case of shared control is better than that of independent control by human and IntelSys.

The control input profiles of human and IntelSys in Fig. 9 (with shared control) and Fig. 7 (without shared control) are different. In the case of shared control, through the final control input, the next state of the inverted pendulum is selected in such a way that the conflict state value would come closer to zero. The effect of the fusion system on the human and IntelSys behavior is clearly seen in these profiles.

Use case 2: IntelSys control model mismatches with that used for conflict model but its control inputs are admissible. Human control model parameters are same as in Use Case 1. IntelSys control model parameters (used in conflict model): $Q_{r 1}=2 I, R_{r 1}=50$. IntelSys control model parameters (actual): $Q_{r 2}=20 I, R_{r 2}=6$. The simulation results for this use case are shown in Figures 10,11 and 12. The shared controller successfully resolves the conflict and brings the inverted pendulum to the zero state. The shared controller uses actual conflict state value.

Use case 3: In this use case, human control inputs are inadmissible while that of IntelSys are admissible. Hence, predicted conflict state is used to compute the final control input instead of actual conflict state. Inadmissibility is introduced by inverting the sign of human LQR optimal gain and hence that of control input. Simulation results are shown in Figures 13, 14 and 15.

Comparing the control input profiles of human in the case of shared control and individual control, it can be seen that the shared controller through its closed loop control manages the human control input in such a way that the inverted pendulum should remain stable and come to a zero state. This is possible because the shared controller uses the predicted conflict state $\hat{X}_{d}$ instead of actual conflict state $X_{d}$ for the calculation of final control input. Thus, it ignores the inadmissible human behavior.
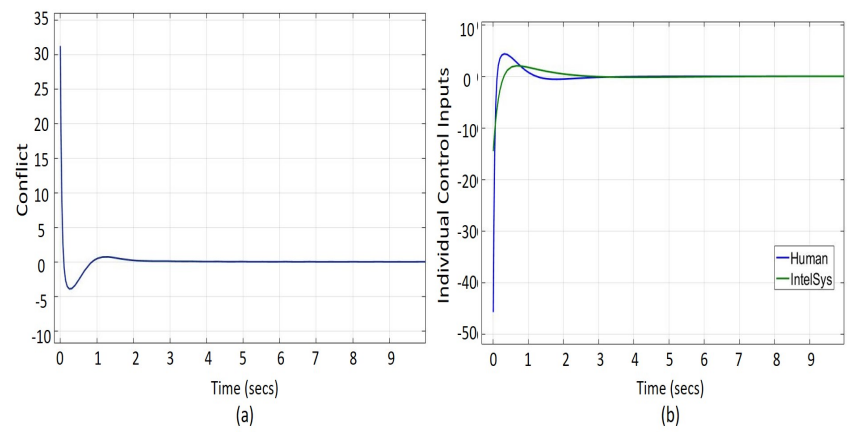

Fig. 7. Use Case 1: (a) Conflict (Actual) Profile. The conflict value is reduced to zero and remains steady at the zero level. The time required for the conflict state to come to zero is dependent on the $\mathrm{Q}$ and $\mathrm{R}$ matrices used by the Shared controller.
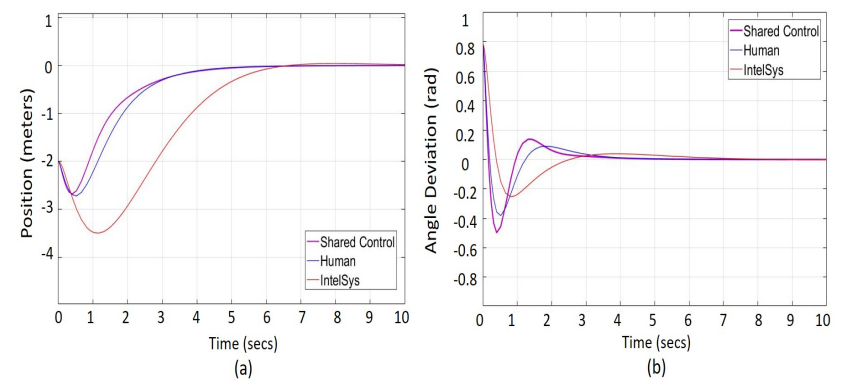

Fig. 8. Use Case 1: Position (state) Profiles (a) and angle deviation (state) Profiles (b) for the cases of shared control and independent control by human and IntelSys. State profile is improved in the case of shared control, (b) Individual control Inputs of human and IntelSys in the case of independent control.
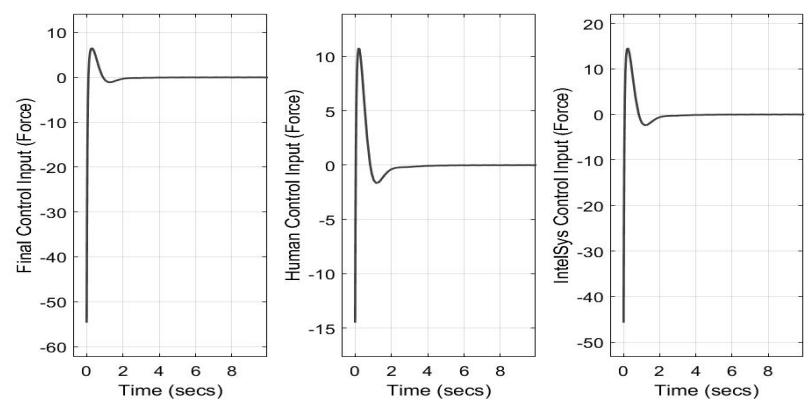

Fig. 9. Use Case 1: Shared Control input profiles of human, IntelSys and fusion system (final control input). Similarity in the profiles of human and IntelSys is due to the conflict resolution

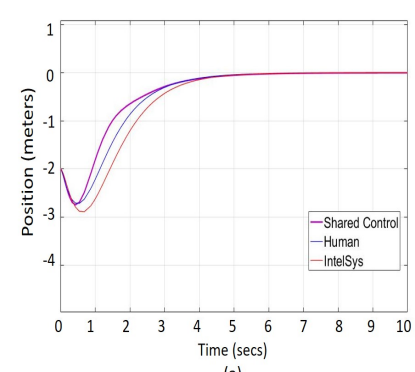

(a)

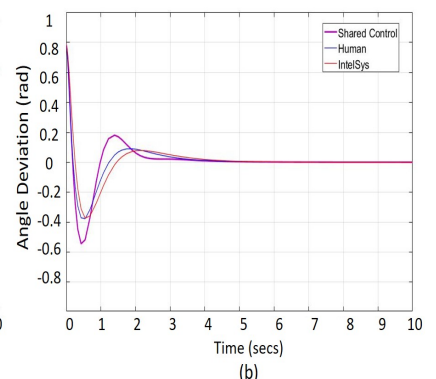

(b)
Fig. 10. Use Case 2: Position (state) (a) and angle deviation (state) (b) profiles for the cases of shared and independent control by human and IntelSys. 

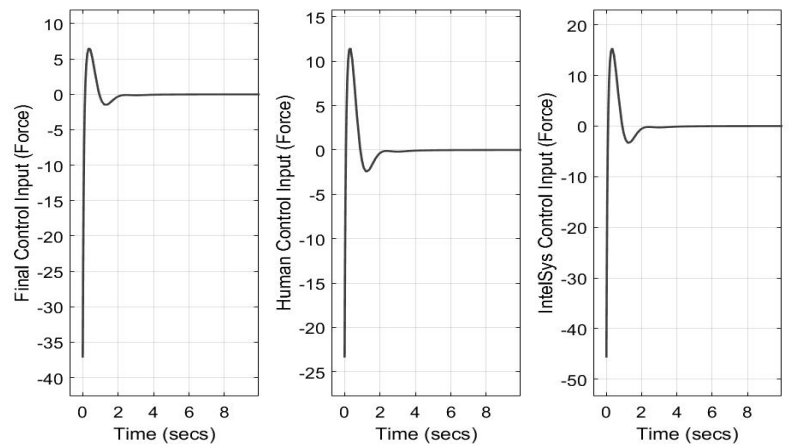

Fig. 11. Use Case 2: Shared Control input profiles of human, IntelSys and fusion system (final control input).
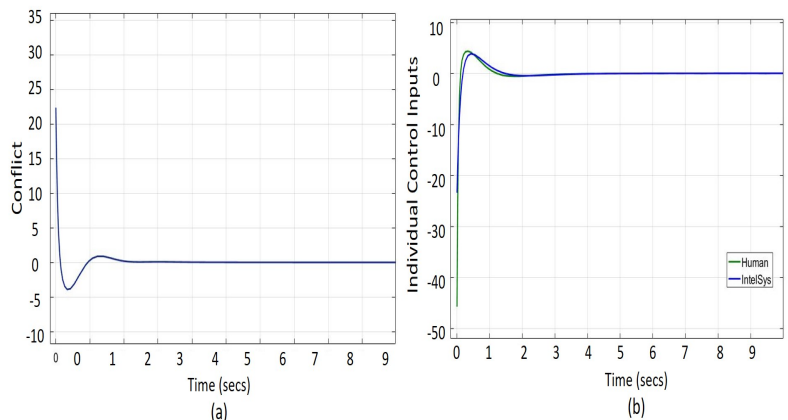

Fig. 12. Use Case 2: Conflict (Actual) Profile (a), Individual control Inputs of human and IntelSys in the case of independent control (b).

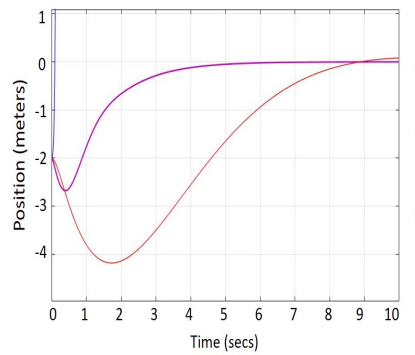

(a)

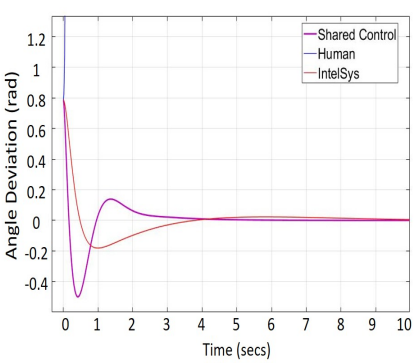

Fig. 13. Use Case 3: Position (state) (a) and angle deviation (state) (b) profiles for the cases of shared and independent control by human and IntelSys.
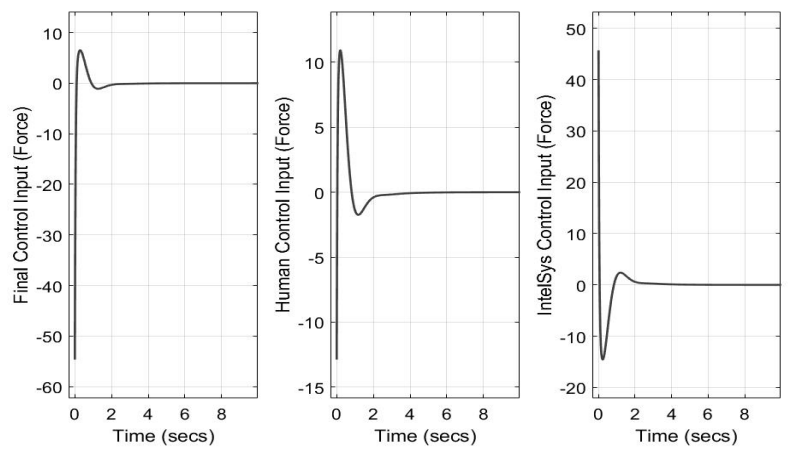

Fig. 14. Use Case 3: Shared Control input profiles of human, IntelSys and fusion system (final control input).

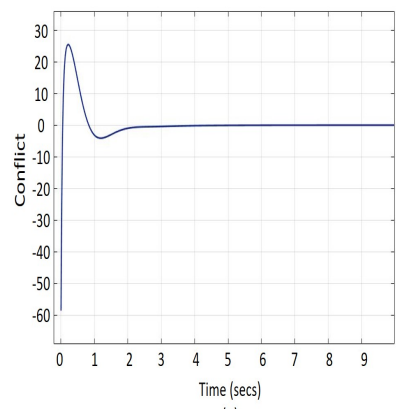

(a)

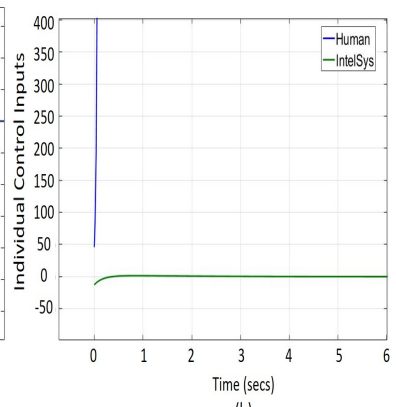

(b)
Fig. 15. Use Case 3: Conflict (Actual) Profile (a), Individual control Inputs of human and IntelSys in the case of independent control (b).

\section{CONCLUSIONS}

Fusion was performed via conflict resolution using adaptive LQR control. Inadmissible control inputs from human/IntelSys were viewed as a inadmissible individual control strategy. This allowed to define all the use cases where the human and IntelSys control strategies/inputs with respect to its admissibility. Simulated results show that the presented shared control strategy works for all the types of use cases.

As a part of the future work, a detailed and complex application based on this framework needs to be developed for the human-intelligent shared driving. Along with this application, the framework has to be enhanced to make it more human centric (without loss of performance) and to make it more robust with respect to nonlinear model uncertainties.

\section{REFERENCES}

[1] F. Ferraguti et al., "A two-layer approach for shared control in semiautonomous IntelSys surgery", 2015 European Control Conference (ECC), Linz, 2015, pp. 747-752.

[2] J. Jiang and A. Astolfi, "Shared-control for a UAV operating in the 3D space", 2015 European Control Conference (ECC), Linz, 2015, pp. $1633-1638$

[3] H. Maske, G. Chowdhary and P. Pagilla, "Intent aware shared control in off-nominal situations", 2016 IEEE 55th Conference on Decision and Control (CDC), Las Vegas, NV, 2016, pp. 5171-5176.

[4] Y. Li, K. P. Tee, W. L. Chan, R. Yan, Y. Chua and D. K. Limbu, "Continuous Role Adaptation for Human-IntelSys Shared Control", in IEEE Transactions on IntelSysics, vol. 31, no. 3, pp. 672-681, June 2015.

[5] S. Y. Jiang, C. Y. Lin, K. T. Huang and K. T. Song, "Shared Control Design of a Walking-Assistant IntelSys", in IEEE Transactions on Control Systems Technology, vol. 25, no. 6, pp. 2143-2150, Nov. 2017.

[6] S. Mosbach, M. Flad and S. Hohmann, "Cooperative longitudinal driver assistance system based on shared control", 2017 IEEE International Conference on Systems, Man, and Cybernetics (SMC), Banff, AB, 2017, pp. 1776-1781.

[7] T. Wada, K. Sonoda, T. Okasaka and T. Saito, "Authority transfer method from automated to manual driving via haptic shared control", 2016 IEEE International Conference on Systems, Man, and Cybernetics (SMC), Budapest, 2016, pp. 002659-002664.

[8] C. B. Chng, Y. Ho and C. K. Chui, "Automation of retinal surgery: A shared control IntelSys system for laser ablation", 2015 IEEE International Conference on Information and Automation, Lijiang, 2015, pp. 1957-1962.

[9] H. Aschemann, R. Prabel and D. Schindele, "Hysteresis compensation and adaptive LQR design for an electro-pneumatic clutch for heavy trucks", 2013 European Control Conference (ECC), Zurich, 2013, pp. 1475-1480. 